\title{
446.
}

\section{ON THE MECHANICAL DESCRIPTION OF A NODAL BICIRCULAR QUARTIC.}

[From the Proceedings of the London Mathematical Society, vol. III. (1869-1871), pp. 100-106.]

The ingenious method, devised by Mr S. Roberts (Proceedings, vol. II. p. 133) for the description of a nodal bicircular quartic suggests a further investigation. We have a quadrilateral $O A A^{\prime} O^{\prime}$, in which the adjacent sides $O A, A A^{\prime}$ are equal to each other, and the other two adjacent sides $O O^{\prime}, O^{\prime} A^{\prime}$ are also equal to each other; $O, O^{\prime}$ are fixed points; and we have thus a link $A A^{\prime}$, the extremities of which are connected

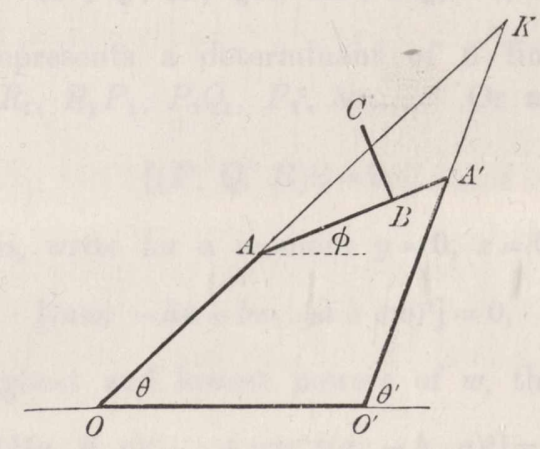

with the radii $O A, O^{\prime} A^{\prime}$ respectively, and consequently describe circles about the centres $O, O^{\prime}$ respectively, the radius $O A$ of the one circle being equal to the length $A A^{\prime}$ of the link, and the radius $O^{\prime} A^{\prime}$ of the other circle being equal to the distance $O O^{\prime}$ of the centres. The theorem is, that any point $C$, rigidly connected with the $\operatorname{link} A A^{\prime}$, describes a nodal bicircular quartic, that is, a quartic curve with three nodes (or unicursal quartic), two of the nodes being the circular points at infinity. Any such curve is the inverse of a conic, and it is also the antipode of a conic; viz., if at each 
point of the curve we draw a line at right angles to the radius vector from the node, these lines envelope a conic having for its pedal the curve in question. It is worth noticing at the outset that to a given position of $A^{\prime}$ there correspond two positions of $A$, viz., the broken line $O A A^{\prime}$ may occupy two positions situate symmetrically on the opposite sides of the line $O A^{\prime}$. But to a given position of $A$, there corresponds only one position of $A^{\prime}$; viz., the broken line $A A^{\prime} O^{\prime}$ is situate symmetrically with $A O O^{\prime}$ on the opposite side of the axis of symmetry $O^{\prime} A$; the only other position would be $A^{\prime}$ coinciding with $O$, that is, $A A^{\prime}$ with $A O$, and the locus of $C$ would then be a circle. If the equalities $O A=A A^{\prime}, O^{\prime} A^{\prime}=O O^{\prime}$ did not subsist, then to a given position of $A^{\prime}$ there would correspond two positions of $A$, and to a given position of $A$ two positions of $A^{\prime}$, and the locus of $C$ would be of a higher order than in the actual problem.

I have called $A A^{\prime}$ the link; $O O^{\prime}$ may be called the bar. $O A$ is then the linkradius, $O^{\prime} A^{\prime}$ the bar-radius; moreover $A A^{\prime} C$ may be called the constant triangle; and, producing $O A, O^{\prime} A^{\prime}$ to meet in $K$, then $A A^{\prime} K$ may be called the variable triangle. Since at any instant the motion of $A$ is normal to $K A$, and the motion of $A^{\prime}$ normal to $K A^{\prime}$, it is clear that the motion at that instant of the constant triangle is a motion of rotation about the point $K$.

Imagine any two positions of the link; say these are $A_{1} A_{1}^{\prime}$, and $A_{2} A_{2}^{\prime}$. Join $A_{1} A_{2}$, and at its mid-point draw a perpendicular thereto; join in like manner $A_{1}^{\prime} A_{2}^{\prime}$, and at its mid-point draw a perpendicular thereto; and let these two perpendiculars meet in $\Gamma$; we have the two equal triangles $A_{1} A_{1}^{\prime} \Gamma, A_{2} A_{2}^{\prime} \Gamma$ (viz., $\Gamma A_{1}=\Gamma A_{2}, \Gamma A_{1}^{\prime}=\Gamma A_{2}^{\prime}$, $A_{1} A_{1}{ }^{\prime}=A_{2} A_{2}$ ) with the common vertex $\Gamma$, and which may be brought to coincide with each other by a finite rotation about this point $\Gamma$. Considering any particular given position of $\Gamma$, if we take the constant triangle $A A^{\prime} C$ equal to $A_{1} A_{1}^{\prime} \Gamma$ or $A_{2} A_{2}^{\prime} \Gamma$ (viz., $A C=A_{1} \Gamma, A^{\prime} C=A_{1}^{\prime} \Gamma$ ), then the constant triangle $A A^{\prime} C$ will, in the course of its motion, come at two different times to coincide with the triangles $A_{1} A_{1}{ }^{\prime} \Gamma$ and $A_{2} A_{2}^{\prime} \Gamma$ respectively; that is, $\Gamma$ will be a node on the locus described by the point $C$; and moreover, if $K_{1}$ and $K_{2}$ be the corresponding positions of $K$, then by what precedes, the directions of the motion (or tangents at the node) will be normal to $K_{1} \Gamma$ and $K_{2} \Gamma$ respectively.

It is to be observed that the point $\Gamma$ is determined by means of two arbitrary positions $A_{1} A_{1}^{\prime}, A_{2} A_{2}^{\prime}$ of the link; that is, the position of $\Gamma$ depends upon two arbitrary parameters, and therefore $\Gamma$ may be any point whatever in the plane; if, for an assumed position of $\Gamma$, the two positions $A_{1} A_{1}^{\prime}, A_{2} A_{2}^{\prime}$ of the link are real, then $\Gamma$ is a crunode on the locus; but if imaginary, then $\Gamma$ is an acnode on the locus. The transition case is when the two positions $A_{1} A_{1}{ }^{\prime}, A_{2} A_{2}{ }^{\prime}$, coincide with each other, $\Gamma$ being in this case a cusp on the locus. But from the foregoing general construction for $\Gamma$, it appears that when $A_{1} A_{1}^{\prime}$ and $A_{2} A_{2}^{\prime}$ coincide, $\Gamma$ is in fact the point $K$, the vertex of the variable triangle. I find that the locus of $K$ is a nodal bicircular quartic, symmetrical in regard to the axis $O O^{\prime}$, and having the point $O$ for a node; viz., when, as in the figure, $A A^{\prime}$ is $\angle O O^{\prime}$, then the point $O$ is an acnode, but when $A A^{\prime}$ is $>O O^{\prime}$, then the point $O$ is a crunode. The curve in question-say the "cuspidal locus"- 
is a curve such that any point whatever thereof is a cusp on the curve described by some point $C$; it separates those points $\Gamma$, such that each of them is a crunode on the curve described by some point $C$, from the points $\Gamma$ which are such that each of them is an acnode of the curve, described by some point $C$. If (as in the figure) $A A^{\prime}$ is $\left\langle O O^{\prime}\right.$, then the cuspidal curve is a closed curve (the inverse of an ellipse), the interior region being crunodal, and the exterior region acnodal. If $A A^{\prime}$ is $>O O^{\prime}$, then the cuspidal curve is a figure of eight (inverse of a hyperbola), the two interior regions being crunodal, and the exterior region acnodal.

Passing now to the analytical investigation, I take the origin at $O$, the axis of $x$ being in the direction from $O$ to $O^{\prime}$, and the axis of $y$, at right angles thereto, upwards from $O$. The inclinations of $O A, A A^{\prime}, O^{\prime} A^{\prime}$ to the axis $O x$, are taken to be $\theta, \phi, \theta^{\prime}$ respectively. I write also $O A=A A^{\prime}=a$, and $O O^{\prime}=O^{\prime} A^{\prime}=a^{\prime}$; and

$$
m=\frac{a^{\prime}-a}{a^{\prime}+a},
$$

or, what is the same thing,

$$
m: 1: 1+m: 1-m=a^{\prime}-a: a^{\prime}+a: 2 a^{\prime}: 2 a ;
$$

and finally $A B=b, B C=c$.

Observing that the angle $A A^{\prime} O^{\prime}$ is $=\theta$, we have $\theta^{\prime}=\theta+\phi$; and then, in the quadrilateral $O A A^{\prime} O^{\prime}$, the angles $A, O^{\prime}$ are $=\pi-\theta+\phi, \pi-\theta-\phi$ respectively; whence, projecting on the diagonal $O A^{\prime}$, we have

$$
a \cos \frac{1}{2}(\theta-\phi)=a^{\prime} \cos \frac{1}{2}(\theta+\phi),
$$

which, attending to the value of $m$, is

whence, writing

we have

$$
\tan \frac{1}{2} \theta \tan \frac{1}{2} \phi=m
$$

$$
\tan \frac{1}{2} \theta=u
$$

$$
\tan \frac{1}{2} \phi=\frac{m}{u},
$$

and the sines and cosines of the angles $\theta, \phi, \theta^{\prime}$ can be all of them expressed in terms of the single parameter $u$.

For the locus of $C$ we have

$$
\begin{aligned}
& x=a \cos \theta+b \cos \phi-c \sin \phi, \\
& y=a \sin \theta+b \sin \phi+c \cos \phi,
\end{aligned}
$$

or, instead of $\theta, \phi$ introducing $u$, we have

$$
\left.\begin{array}{l}
x=-a \frac{u^{2}-1}{u^{2}+1}+b \frac{u^{2}-m^{2}}{u^{2}+m^{2}}-c \frac{2 m u}{u^{2}+m^{2}} \\
y=a \frac{2 u}{u^{2}+1}+b \frac{2 m u}{u^{2}+m^{2}}+c \frac{u^{2}-m^{2}}{u^{2}+m^{2}}
\end{array}\right\},
$$


which, in fact, show that the locus is a bicircular quartic. To put in evidence the third node, I assume that the values belonging thereto are $u=u_{1}, u=u_{2}$, and that the coordinates of the node are $\alpha, \beta$; we have thus

$$
\begin{aligned}
& \alpha=-a \frac{u_{1}^{2}-1}{u_{1}^{2}+1}+b \frac{u_{1}^{2}-m^{2}}{u_{1}^{2}+m^{2}}-c \frac{2 m u_{1}}{u_{1}^{2}+m^{2}},=-a \frac{u_{2}^{2}-1}{u_{2}^{2}+1}+b \frac{u_{2}^{2}-m^{2}}{u_{2}^{2}+m^{2}}-c \frac{2 m u_{2}}{u_{2}^{2}+m^{2}}, \\
& \beta=a \frac{2 u_{1}}{u_{1}^{2}+1}+b \frac{2 m u_{1}}{u_{1}^{2}+m^{2}}+c \frac{u_{1}^{2}-m^{2}}{u_{1}^{2}+m^{2}},=-a \frac{2 u_{2}}{u_{2}^{2}+1}+b \frac{2 m u_{2}}{u_{2}^{2}+m^{2}}+c \frac{u_{2}^{2}+m^{2}}{u_{2}^{2}+m^{2}} .
\end{aligned}
$$

These give $b, c, \alpha, \beta$ in terms of $a, m, u_{1}, u_{2}$; and we may then express the values of $x-\alpha, y-\beta$ in terms of $a, m, u_{1}, u_{2}, u$. I find

and then

$$
\begin{aligned}
& b=\frac{a}{m}\left\{-1+\frac{m+1}{\left(u_{1}^{2}+1\right)\left(u_{2}^{2}+1\right)}\left[\left(u_{1}+u_{2}\right)^{2}+(1-m)\left(1-u_{1} u_{2}\right)\right]\right\}, \\
& c=\frac{a}{m}\left\{\quad \frac{m+1}{\left(u_{1}^{2}+1\right)\left(u_{2}^{2}+1\right)}\left[-\left(u_{1}+u_{2}\right)\left(m-u_{1} u_{2}\right)\right]\right\} \text {; }
\end{aligned}
$$

$$
\begin{aligned}
& x=-a \frac{u^{2}-1}{u^{2}+1}+\frac{a}{m}\left\{-1+\frac{m+1}{\left(u_{1}^{2}+1\right)\left(u_{2}^{2}+1\right)}\left[\left(u_{1}+u_{2}\right)^{2}+(1-m)\left(1-u_{1} u_{2}\right)\right]\right\} \frac{u^{2}-m^{2}}{u^{2}+m^{2}} \\
& +\frac{a}{m}\left\{\quad \frac{m+1}{\left(u_{1}^{2}+1\right)\left(u_{2}^{2}+1\right)}\left[\quad\left(u_{1}+u_{2}\right)\left(m-u_{1} u_{2}\right) \quad\right]\right\} \frac{2 m u}{u^{2}+m^{2}}, \\
& y=a \frac{2 u}{u^{2}+1}+\frac{a}{m}\left\{-1+\frac{m+1}{\left(u_{1}^{2}+1\right)\left(n_{2}^{2}+1\right)}\left[\left(u_{1}+u_{2}\right)^{2}+(1-m)\left(1-u_{1} u_{2}\right)\right]\right\} \frac{2 m u}{u^{2}+m^{2}} \\
& +\frac{a}{m}\left\{\quad \frac{m+1}{\left(u_{1}^{2}+1\right)\left(u_{2}{ }^{2}+1\right)}\left[-\left(u_{1}+u_{2}\right)\left(m-u_{1} u_{2}\right) \quad\right]\right\} \frac{u^{2}-m^{2}}{u^{2}+m^{2}}, \\
& \alpha=\frac{a}{m} \frac{m+1}{\left(u_{1}^{2}+1\right)\left(u_{2}^{2}+1\right)}\left(1-u_{1} u_{2}\right)\left(m+u_{1} u_{2}\right), \\
& \beta=\frac{a}{m} \frac{m+1}{\left(u_{1}^{2}+1\right)\left(u_{2}^{2}+1\right)}\left(u_{1}+u_{2}\right)\left(m+u_{1} u_{2}\right) ;
\end{aligned}
$$

and then

$$
\begin{aligned}
& (x-\alpha)=-\frac{2(m+1) a}{\left(u_{1}^{2}+1\right)\left(u_{2}^{2}+1\right)}\left(u^{2}+1\right)\left(u_{1}\right)\left(u-u_{2}\right) \\
& (y-\beta)=-\frac{2(m+1) a}{\left(u_{1}^{2}+1\right)\left(u_{2}^{2}+1\right)} \frac{\left(u-u_{1}\right)\left(u-u_{2}\right)}{\left(u^{2}+1\right)\left(u^{2}+m^{2}\right)} \times\left[\left(u_{1}+u_{2}\right)\left(u^{2}+m\right)-(1-m)\left(1-u_{1} u_{2}\right) u\right],
\end{aligned}
$$

where, of course, the factors $\left(u-u_{1}\right),\left(u-u_{2}\right)$ indicate the node $(\alpha, \beta)$. We have moreover

so that, writing

$$
(x-\alpha)^{2}+(y-\beta)^{2}=\frac{4(m+1)^{2} a^{2}}{\left(u_{1}^{2}+1\right)\left(u_{2}^{2}+1\right)} \frac{\left(u-u_{1}\right)^{2}\left(u-u_{2}\right)^{2}}{\left(u^{2}+1\right)\left(u^{2}+m^{2}\right)},
$$

$$
\begin{aligned}
& \frac{x-\alpha}{(x-\alpha)^{2}+(y-\beta)^{2}}=-\frac{1}{2(m+1) a} \frac{\left[\left(1-u_{1} u_{2}\right)\left(u^{2}+m\right)+(1-m)\left(u_{1}+u_{2}\right) u\right]}{\left(u-u_{1}\right)\left(u-u_{2}\right)} \\
& \frac{y-\beta}{(x-\alpha)^{2}+(y-\beta)^{2}}=-\frac{1}{2(m+1)} u \frac{\left[\left(u_{1}+u_{2}\right)\left(u^{2}+m\right)-(1-m)\left(1-u_{1} u_{2}\right) u\right]}{\left(u-u_{1}\right)\left(u-u_{2}\right)}
\end{aligned}
$$

C. VII. 
we have the locus as the inverse of a conic. To exhibit it as the antipode of a conic, taking $X, Y$ as current coordinates measured from the node as origin, the equation of the line through a point of the locus, at right angles to the radius vector from the node, is

$$
X(x-\alpha)+Y(y-\beta)-(x-\alpha)^{2}-(y-\beta)^{2}=0 ;
$$

or, substituting for $(x-\alpha),(y-\beta)$ their values, this is

$$
\begin{aligned}
& X\left[\left(1-u_{1} u_{2}\right)\left(u^{2}+m\right)+(1-m)\left(u_{1}+u_{2}\right) u\right] \\
+ & Y\left[\left(u_{1}+u_{2}\right)\left(u^{2}+m\right)-(1-m)\left(1-u_{1} u_{2}\right) u\right]+2(m+1) a\left(u-u_{1}\right)\left(u-u_{2}\right)=0 ;
\end{aligned}
$$

and the antipodal conic is thus the envelope of the line represented by this equation. Putting for shortness

$$
P=X\left(1-u_{1} u_{2}\right)+Y\left(u_{1}+u_{2}\right), \quad Q=X\left(u_{1}+u_{2}\right)-Y\left(1-u_{1} u_{2}\right),
$$

the equation is

$$
u^{2}\{P+2(m+1) a\}+u\left\{(1-m) Q-2(m+1) a\left(u_{1}+u_{2}\right)\right\}+m P+2(m+1) a u_{1} u_{2}=0,
$$

and the equation of the conic therefore is

$$
\text { 4. }\{P+2(m+1) a\}\left\{m P+2(m+1) a u_{1} u_{2}\right\}-\left\{(1-m) Q-2(m+1) a\left(u_{1}+u_{2}\right)\right\}^{2}=0,
$$

so that the conic touches each of the lines $P+2(m+1) a=0, m P+2(m+1) a u_{1} u_{2}=0$ at its intersection with the line $(1-m) Q-2(m+1) a u_{1}=0$. If these lines were constructed, one other condition would suffire for the construction of the conic.

The before-mentioned equations

$$
\begin{aligned}
& \alpha=\frac{a}{m} \frac{m+1}{\left(u_{1}^{2}+1\right)\left(u_{2}^{2}+1\right)}\left(1-u_{1} u_{2}\right)\left(m+u_{1} u_{2}\right), \\
& \beta=\frac{a}{m} \frac{m+1}{\left(u_{1}^{2}+1\right)\left(u_{2}^{2}+1\right)}\left(u_{1}+\bar{u}_{2}\right)\left(m+u_{1} u_{2}\right),
\end{aligned}
$$

give

and thence

$$
\alpha^{2}+\beta^{2}=\frac{a^{2}}{m^{2}} \frac{(m+1)^{2}}{\left(u^{2}+1\right)\left(u_{2}^{2}+1\right)}\left(m+u_{1} u_{2}\right)^{2}
$$

$$
\begin{aligned}
\frac{\alpha}{\alpha^{2}+\beta^{2}} & =\frac{m}{(m+1) a} \frac{1-u_{1} u_{2}}{m+u_{1} u_{2}}, \\
\frac{\beta}{\alpha^{2}+\beta^{2}} & =\frac{m}{(m+1) a} \frac{u_{1}+u_{2}}{m+u_{1} u_{2}},
\end{aligned}
$$

which determine $u_{1}+u_{2}$ and $u_{1} u_{2}$ rationally in terms of $\alpha, \beta$. For the cuspidal curve, writing $u_{1}=u_{2}=v$, we have

$$
\begin{aligned}
\frac{\alpha}{\alpha^{2}+\beta^{2}} & =\frac{m}{(m+1) a} \frac{1-v^{2}}{m+v^{2}}, \\
\frac{\beta}{\alpha^{2}+\beta^{2}} & =\frac{m+1) a}{\left(m+v^{2}\right.},
\end{aligned}
$$


which show that the cuspidal curve is the inverse of a conic (viz., of an ellipse, if, as in the figure, $m$ is positive). The result in the very same form would be obtained by considering the curve as the locus of the vertex $K$ of the variable triangle.

If we imagine a plane rigidly connected with the link $A A^{\prime}$, and carried along with it, then $(b, c)$ are the coordinates of the point $C$ in this moveable plane; and if, as above, $(\alpha, \beta)$ are the coordinates of the node, then $(b, c)$ and also $(\alpha, \beta)$, are given functions of $\left(u_{1}, u_{2}\right)$. We have thus $(b, c)$ functions of $(\alpha, \beta)$, and reciprocally $(\alpha, \beta)$ functions of $(b, c)$; that is, we have a correspondence between the points of the fixed plane and those of the variable plane. It is worth while to investigate the nature of this correspondence, although the result does not appear to be one of any elegance.

Writing

$$
\begin{aligned}
& A=\frac{(m+1) a}{m} \frac{\alpha}{\alpha^{2}+\beta^{2}}, \\
& B=\frac{(m+1) a}{m} \frac{\beta}{\alpha^{2}+\beta^{2}},
\end{aligned}
$$

we may, in place of $(\alpha, \beta)$, consider the point in the fixed plane as given by means of the inverse coordinates $(A, B)$. And then, if $p=u_{1}+u_{2}, q=1-u_{1} u_{2}$, we have

whence

$$
A=\frac{q}{m+1-q}, \quad B=\frac{q}{m+1-q}
$$

Hence

$$
\begin{aligned}
p & =\frac{(m+1) B}{1+A}, \quad q=\frac{(m+1) A}{1+A}, \\
p^{2}+q^{2} & =\left(1+u_{1}^{2}\right)\left(1+u_{2}^{2}\right)=\frac{(m+1)^{2}\left(A^{2}+B^{2}\right)}{(1+A)^{2}} .
\end{aligned}
$$

$$
\begin{aligned}
\frac{m}{a}\left(b+\frac{a}{m}\right) & =\frac{m+1}{p^{2}+q^{2}}\left[p^{2}-(m-1) q\right] \\
-\frac{m}{a} c & =\frac{m+1}{p^{2}+q^{2}} p(m-1+q)
\end{aligned}
$$

which determine $(b, c)$ in terms of $(p, q)$; that is, of $(A, B)$ or of $(\alpha, \beta)$.

In reference to some other constructions given in $\mathrm{Mr}$ Roberts' paper, it may be remarked that if we have a moveable plane $\Pi_{1}$ always coincident with a fixed plane $\Pi$, and if a condition of the motion is that a circle $C_{1}$, fixed in the plane $\Pi_{1}$ and carried along with it, always touches a fixed circle $C$ in the plane $\Pi$, then this same condition may be expressed indifferently in either of the forms-(1) a circle $C_{1}$ in the plane $\Pi_{1}$ always passes through a fixed point of $\Pi$; (2) a point in the plane $\Pi_{1}$ is always situate on a fixed circle $C$ in the plane $\Pi$. But if either of the circles $C, C_{1}$ reduce itself to a line, then we have two distinct forms of condition; viz., first, if a fixed line $L_{1}$ in the plane $\Pi_{1}$ always touches a fixed circle $C$ in the plane $\Pi$, this is equivalent to the condition that a fixed line $L_{1}$ in the plane $\Pi_{1}$ always passes 
through a fixed point of the plane $\Pi$. And secondly, if a fixed circle $C_{1}$ in the plane $\Pi_{1}$ always touches a fixed line $L$ in the plane $\Pi$, this is equivalent to the condition that a fixed point in the plane $\Pi_{1}$ is always situate in a fixed line $L_{1}$ in the plane $\Pi_{1}$. The different forms of condition therefore are :

(a) A fixed circle $C_{1}$ in the plane $\Pi_{1}$ always touches a fixed circle $C$ in the plane $\Pi$ (where, as above, either circle indifferently may be reduced to a point).

$(\beta)$ A fixed line $L_{1}$ in the plane $\Pi_{1}$ always passes through a fixed point $C$ in the plane $\Pi$.

( $\gamma$ ) A fixed point $C_{1}$ in the plane $\Pi_{1}$ is always situate in a fixed line $L$ of the plane II.

Hence, if the motion of the plane $\Pi_{1}$ satisfy any two such conditions (of the same form or of different forms, viz., the conditions may be each $\alpha$, or they may be $\alpha$ and $\beta$, \&c.), then the motion of the plane $\Pi_{1}$ will depend on a single variable parameter, and the question arises as to the locus described by a given point, or enveloped by a given line, of the plane $\Pi$; and again of the locus traced out, or enveloped, on the moving plane $\Pi_{1}$ by a given point of the plane $\Pi$. The case considered in the present paper is of course a particular case of the two conditions being each of them of the form $\alpha$.

It may be remarked, that if the two conditions be each of them $\beta$, then there will be in the plane $\Pi_{1}$ a fixed point $C_{1}$ which describes a circle; and similarly, if the two conditions be each of them $\gamma$, then there will be in the plane $\Pi_{1}$ a fixed point $C_{1}$ which describes a $\operatorname{circle}\left({ }^{1}\right)$; that is, the combination $\beta \beta$ is a particular case of $\alpha \beta$, and the combination $\gamma \gamma$ a particular case of $\alpha \gamma$.

1 The theorem is, that if an isosceles triangle, on the base $A A^{\prime}$ and with angle $=2 \omega$ at the vertex $C$, slide between two lines $O A, O A^{\prime}$ inclined to each other at an angle $\omega$, in such manner that $C$ is the centre of the circle circumscribed about $O A A^{\prime}$, then the locus of $C$ is a circle having $O$ for its centre. 